\title{
CARACTERÍSTICAS BIOMÉTRICAS DE MUDAS DE Tabebula Chrysotricha (STANDL.) FORMADAS EM DIFERENTES SUBSTRATOS E SOLUÇÕES DE FERTIRRIGAÇÃO, QUANDO PLANTADAS EM CAMPO ${ }^{1}$
}

\author{
Isabele Sarzi², Roberto Lyra Villas Boas², Magali Ribeiro da Silva ${ }^{3}$ e José Luis de Carvalho ${ }^{4}$
}

\begin{abstract}
RESUMO - Este trabalho objetivou estudar as características biométricas de plantas de ipê-amarelo (Tabebuia chrysotricha Standl.) formadas, na fase de viveiro, em função de quatro substratos, variando as soluções de fertirrigação. Para compor os substratos, foram utilizadas fibras de coco fibrosa e granulada, formando os tratamentos $100 \%$ fibrosa, $60 \%$ fibrosa e $40 \%$ granulada, $40 \%$ fibrosa e $60 \%$ granulada e $100 \%$ granulada. A adubação de base foi igual em todos os tratamentos, e as soluções de adubação variaram, a fim de se obterem soluções completas com condutividades elétricas de $1,06 \mathrm{dS} \mathrm{m}^{-1} ; 2,12 \mathrm{dS} \mathrm{m}^{-1}$; 3,2 dS m-1; e 4,24 dS ms. As sementes foram colocadas diretamente nos tubetes $(120 \mathrm{~mL})$ contendo os respectivos substratos e receberam as fertirrigações por subsuperfície uma vez por semana, respeitando-se os tratamentos de adubação. Quando as mudas atingiram aproximadamente $20 \mathrm{~cm}$ de altura, elas foram realocadas sob tela de $9 \%$ de sombreamento, onde permaneceram até o plantio, em Taubaté, SP. Mudas produzidas em fibra de coco $100 \%$ granulada alcançaram, em campo, maiores alturas, diâmetros de coleto e número de folhas. Apesar das diferentes soluções de fertirrigação aplicadas, as alturas de parte aérea se igualaram a partir dos 167 dias após o plantio em campo. Recomendase a produção de mudas de ipê-amarelo ( $T$. chrysotricha) em substratos contendo fibra de coco granulada e soluções de fertirrigação com condutividade elétrica de 1,06 dS m-1.
\end{abstract}

Palavras-chave: Substrato, T. chrysotricha e Sobrevivência em campo.

\section{BIOMETRIC CHARACTERIZATIONS OF Tabebula Chrysotricha (STANDL.) SEEDLINGS FORMED IN DIFFERENT SUBSTRATES AND FERTIRRIGATION SOLUTIONS, WHEN PLANTED IN FIELDS}

\begin{abstract}
The work aimed to study the formation of Tabebuia chrysotricha Standl. seedlings in function of four substrates, varying the fertirrigation solutions. To compose the substrate, fibrous and granulated coconut fiber was used, obtaining the following treatments: $100 \%$ fibrous, $60 \%$ fibrous and $40 \%$ granulated, $40 \%$ fibrous and $60 \%$ granulated and $100 \%$ granulated. The base fertilization was the same for all treatments and the solutions of fertilization varied in order to obtain complete solutions with electric conductivities of $1.06 \mathrm{dS} \mathrm{m}^{-1}, 2.12 \mathrm{dS} \mathrm{m}^{-1}, 3.2 \mathrm{dS} \mathrm{m}^{-1}$ and $4.25 \mathrm{dS} \mathrm{m}^{-1}$. The propagative material was sowed directly into plastic containers $(120 \mathrm{~mL})$ with the respective substrates. The fertilization was received through sub irrigation once a week, respecting the treatments of fertilizations. When the seedlings reached $20 \mathrm{~cm}$ of height, approximately, they were replaced under black screens (9\% of shading), where they remained until planting in Taubaté-SP. Seedlings produced in coconut fiber $100 \%$ granulate reached, in the field, higher heights, stem diameters and number of leaves. In function of applied solutions, heights of the aerial part were homogeneous starting from 167 days after planting. The production of T. chrysotricha seedlings is recommended in granulated coconut fiber substrate and fertilizer solutions with electric conductivity of $1.06 \mathrm{dS} \mathrm{m}^{-1}$.
\end{abstract}

Keywords: Substrate, T. Chrysotricha and Survival of the seedlings.

\footnotetext{
${ }^{1}$ Recebido em 18.08.2008 e aceito para publicação em 14.10.2009.

${ }^{2}$ Instituto Florestal do Estado de São Paulo, IF, Brasil. E-mail: <isarzi@iflorestal.sp.gov.br>e <rlvboas@fca.unesp.br>.

${ }^{3}$ Universidade Estadual Paulista Júlio de Mesquita Filho, UNESP, Brasil. E-mail: <magaliribeiro@fca.unesp.br>.

${ }^{4}$ Universidade Federal do Rio de Janeiro, UFRJ, Brasil.
} 


\section{INTRODUÇÃO}

O ipê-amarelo-cascudo (Tabebuia chrysotricha Standl.) é uma Bignoniaceae de pequeno porte, crescimento rápido e ciclo de vida curto, heliófita, seletiva higrófita. Ocorre associada a diferentes formações florestais desde o Nordeste ao Sul do Brasil, mas raramente se encontram indivíduos dessa espécie no interior da floresta primária. A árvore é extremamente ornamental, principalmente, quando em flor. É a espécie de ipê-amarelo mais cultivada em praças e ruas de cidades. É particularmente útil para arborização de ruas estreitas e sob redes elétricas em virtude de seu pequeno porte. Sua grande importância está, também, na plasticidade ecológica, crescimento rápido e produção de frutos para a avifauna, tornando-a prioritária para recuperação de áreas degradadas, especialmente, matas ciliares (LORENZI, 1992).

A madeira é moderadamente pesada, resistente, difícil de serrar, de grande durabilidade, mesmo quando em condições adversas. É própria para obras externas como postes, peças para pontes, tábuas para cercas, currais e haras, para obras internas em construção civil, como tacos e tábuas para assoalho, rodapés, molduras, entre outros (LORENZI, 1992).

A produção de mudas florestais, em qualidade e quantidade, é uma das fases mais importantes para o estabelecimento de bons povoamentos florestais. Várias pesquisas científicas e avanços técnicos têm sido realizados com o objetivo de melhorar a qualidade das mudas, assegurando boa adaptação e crescimento após o plantio (GONÇALVES et al., 2005).

Leite et al. (2005) consideraram que, em um projeto de reflorestamento, a qualidade das mudas é muito importante, por estar relacionada diretamente com a qualidade do povoamento e, consequentemente, da colheita final. Por se tratar de investimentos de longo prazo, o rigor torna-se maior, justificando o dispêndio com o controle contínuo da qualidade.

Na produção de mudas, são necessários, basicamente, o substrato, o recipiente para acondicioná-lo e os nutrientes. Nos viveiros de alta rotatividade, em geral, utilizam-se tubetes de polipropileno de, aproximadamente, $120 \mathrm{~cm}^{3}$ de capacidade e substratos constituídos, principalmente, por resíduos orgânicos (MORAES NETO et al., 2003). O uso de resíduos orgânicos florestais, como componente de substratos para a produção de mudas, é prática comum em empresas de papel e celulose, sendo que, de modo geral, a casca de Pinus e de outras espécies florestais é o resíduo mais utilizado (MAIA, 1999).

O tipo de substrato e o tamanho do recipiente são os primeiros aspectos que devem ser pesquisados para se garantir a produção de mudas de boa qualidade. O tamanho do recipiente deve ser tal que permita o desenvolvimento da raiz sem restrições durante o período de permanência no viveiro. O substrato exerce influência marcante na arquitetura do sistema radicular e no estado nutricional das plantas, afetando, profundamente, a qualidade das mudas (CARVALHO FILHO et al., 2003). As más formações causadas nas raízes pelos recipientes, na fase de mudas, podem provocar problemas nas plantas adultas muitos anos depois. Paulino et al. (2003), estudando a distribuição do sistema radicular de árvores de acácia-negra (Acacia mearnsii) com 3 anos de idade, provenientes de mudas formadas em diferentes recipientes, observaram que o laminado de madeira apresentou-se superior ao tubete quanto ao comprimento de raízes nas linhas de plantio e que raízes cresceram melhor onde as condições de densidade e porosidade do solo se encontravam mais adequadas.

A prática da fertilização em viveiros florestais é importante para que as mudas cresçam rapidamente, vigorosas, resistentes, rústicas e bem nutridas. Assim, apresentarão as qualidades necessárias para suportarem as condições adversas do campo após o plantio (LOPES, 2002). Diferentes grupos sucessionais de espécies de árvores apresentam variações no padrão comportamental relacionadas à capacidade de aquisição, concentração e eficiência de utilização de nutrientes. As espécies pioneiras tendem à maior eficiência em absorver e utilizar os nutrientes, quando comparadas com as espécies secundárias e climácicas, além do comportamento distinto entre espécies dentro do mesmo grupo sucessional (RESENDE et al., 2000).

Segundo Cruz et al. (2004), a variação nas características dos substratos tem dificultado, de maneira considerável, a recomendação de adubação, pois a capacidade de retenção de umidade e de nutrientes difere de acordo com os componentes e a proporção entre eles. Os referidos autores relataram que os problemas nutricionais são muito comuns, tanto pela falta quanto pelo excesso ou desequilíbrio entre nutrientes. 
A falta de informações a respeito dos requerimentos nutricionais das espécies florestais nativas conduz à necessidade de realização de ensaios para obtenção de informações mais precisas para que se possam produzir mudas de melhor qualidade (CRUZ et al., 2004).

Este experimento teve por objetivo estudar as características biométricas pós-plantio de plantas de ipê-amarelo quando produzidas em diferentes substratos e diferentes soluções de fertirrigação na fase de muda.

\section{MATERIAL E MÉTODOS}

A produção das mudas foi conduzida no Viveiro do Departamento de Recursos Naturais/Ciências Florestais, na Fazenda Experimental Lageado, da Faculdade de Ciências Agronômicas da UNESP de Botucatu, SP, e no Viveiro Florestal da Capital, situado no Parque Estadual Alberto Löefgren, Instituto Florestal de São Paulo. As mudas prontas para expedição foram plantadas em campo no Município de Taubaté, em área do Instituto Florestal de São Paulo.

O experimento foi conduzido no período de setembro de 2004 a maio de 2006. As mudas foram formadas em oito meses e acompanhadas em campo por 12 meses após o plantio.

Foram testados 16 tratamentos para a produção de mudas de T. chrysotricha (Tabela 1). Para formar os tratamentos, seguiu-se o esquema fatorial de $4 \times 4$, sendo quatro substratos à base de fibra de coco (100\% fibrosa; $60 \%$ fibrosa $+40 \%$ granulada; $40 \%$ fibrosa $+60 \%$ granulada e $100 \%$ granulada) e quatro soluções de fertirrigação (1,06 dS m ${ }^{-1} ; 2,12 \mathrm{dS} \mathrm{m}^{-1} ; 3,2 \mathrm{dS} \mathrm{m}^{-1}$ e 4,24 dS m¹). Cada tratamento contou com quatro blocos de 50 unidades, inteirando 3.200 mudas, sendo plantadas quatro mudas por bloco, completando 256 mudas plantadas.
As misturas dos substratos foram realizadas com base volumétrica, com a fibra de coco previamente umedecida, segundo as prescrições do fabricante.

Depois de realizadas as misturas, os substratos foram espalhados em uma superfície lisa e plana onde receberam a adubação de base, seguindo a recomendação de Gonçalves et al. (2005): -150 g de N (sulfato de amônio) (24\% S-SO ${ }_{4}^{2-;}$; $1 \%$ N- $\mathrm{NH}_{4}^{-}$); -300 g de $\mathrm{P}_{2} \mathrm{O}_{5}$ (superfosfato

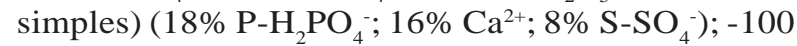
g de $\mathrm{K}_{2} \mathrm{O}$ (cloreto de potássio) (52\% K+; 47\% Cl-); -150 g de "fritas" (FTE BR 12) (9\% Zn; 1,8\% B; 0,8\% $\mathrm{Cu}$; 2\% Mn; 3,5\% Fe; e 0,1\% Mo).

Os tubetes $(120 \mathrm{~mL})$ dos tratamentos com 100\% de fibra de coco fibrosa foram preenchidos um a um, pois a textura do substrato não permitiu a fluidez do material. Os tubetes dos demais tratamentos foram preenchidos colocando-se o respectivo substrato sobre eles e procedendo-se batimento manual, para acomodação do material, repetindo-se o procedimento até completar o tubete e, retirando-se o substrato em excesso.

Para compor os tratamentos de diferentes condutividades elétricas, utilizaram-se soluções completas com concentrações diferentes, a fim de apresentarem condutividades elétricas crescentes, a saber: $1,06 \mathrm{dS} \mathrm{m}^{-1}, 2,12 \mathrm{dS} \mathrm{m}^{-1}$, $3,20 \mathrm{dS} \mathrm{m}^{-1}$ e $4,24 \mathrm{dS} \mathrm{m}^{-1}$.

Utilizou-se como base para a solução completa de adubação a formulação usada por um viveiro florestal comercial, localizado em Ibaté, SP, com condutividade elétrica de 2,12 dS m-1. Para $100 \mathrm{~L}$ de água: - 42,6 g de nitrato de cálcio (19\% $\left.\mathrm{Ca}^{2+} ; 15 \% \mathrm{~N}_{-} \mathrm{NO}_{3}^{-} ; 1 \% \mathrm{~N}^{-} \mathrm{NH}_{4}^{-}\right)$; - 15 g de cloreto de potássio (52\% K+; 47\% Cl-); - 7,6 g de monoamônio fosfato (MAP) (11\% N- $\mathrm{NH}_{4}^{-} ; 26 \%$ P- $\mathrm{H}_{2} \mathrm{PO}_{4}^{-}$); - 50 g de sulfato de amônio (24\% S-SO ${ }_{4}^{2-}$ ; $21 \% \mathrm{~N}-\mathrm{NH}_{4}^{-}$); e - 32,6 g de sulfato de magnésio $\left(13 \% \mathrm{~S}^{-\mathrm{SO}_{4}}{ }^{2-} ; 10 \% \mathrm{Mg}^{2+}\right.$ ).

Tabela 1-Tratamentos usados na formação de mudas de ipê-amarelo (T. chrysotricha). Table 1 - Treatments used in the formation of T. chrysotricha seedlings.

\begin{tabular}{|c|c|c|c|c|}
\hline \multirow[t]{2}{*}{ Composição dos Substratos } & \multicolumn{4}{|c|}{ Condutividade elétrica das soluções de fertirrigação } \\
\hline & $1,06 \mathrm{dS} \mathrm{m}^{-1}$ & $2,12 \mathrm{dS} \mathrm{m}^{-1}$ & $3,20 \mathrm{dS} \mathrm{m}^{-1}$ & $4,24 \mathrm{dS} \mathrm{m}^{-1}$ \\
\hline $100 \%$ fc fibrosa & $\mathrm{T} 1$ & $\mathrm{~T} 2$ & T3 & $\mathrm{T} 4$ \\
\hline $60 \%$ fc fibrosa $+40 \%$ fc granulada & T 5 & T 6 & $\mathrm{~T} 7$ & T 8 \\
\hline $40 \%$ fc fibrosa $+60 \%$ fc granulada & Т 9 & $\mathrm{~T} 10$ & $\mathrm{~T} 11$ & $\mathrm{~T} 12$ \\
\hline $100 \%$ fc granulada & $\mathrm{T} 13$ & $\mathrm{~T} 14$ & $\mathrm{~T} 15$ & $\mathrm{~T} 16$ \\
\hline
\end{tabular}

$\mathrm{Fc}=$ fibra de coco; $\mathrm{T}=$ tratamento; $f c=$ coconut fiber $;$ and $\mathrm{T}=$ treatment. 
Para a solução de $1,06 \mathrm{dS} \mathrm{m}^{-1}$, utilizaram-se $50 \%$ das quantidades dos adubos. Para compor a solução de CE de 3,20 dS m-1 , foram utilizados $50 \%$ a mais das quantidades de adubo, enquanto para a solução de CE 4,14 dS $\mathrm{m}^{-1}$ se utilizou o dobro da quantidade.

Em cada tubete preenchido com o substrato, foram colocadas três sementes em 29 de setembro de 2004. Os tubetes foram mantidos em câmara de nebulização com sombreamento de $50 \%$ e umidade relativa do ar de $80 \%$.

Quando as plântulas atingiram $5 \mathrm{~cm}$ de altura, aos 42 dias após a semeadura as bandejas foram levadas para túnel plástico do Viveiro da Faculdade de Ciências Agronômicas da UNESP de Botucatu, quando começaram a receber, semanalmente, as fertirrigações por subsuperfície. As soluções de fertirrigação foram mantidas em contato com os substratos até que se pudesse vê-la na superfície superior dos tubetes. Para os demais dias da semana, as irrigações foram feitas com mangueira, tendo um crivo acoplado na extremidade. Aos 77 dias após a semeadura, as mudas foram transportadas para casa de vidro, com tela de sombreamento de 50\%, do Viveiro Florestal da Capital do Instituto Florestal de São Paulo.

As mudas permaneceram em casa de vidro recoberta com tela de $50 \%$ de sombreamento até alcançarem, aproximadamente, $20 \mathrm{~cm}$ de altura, aos 153 dias após a semeadura.

Aos 218 dias após a semeadura, completou-se o processo de rustificação das mudas, quando foram plantadas em campo em área do Viveiro Florestal de Taubaté. Para isso, prepararam-se, aproximadamente, $1.500 \mathrm{~m}^{2}$ de área para o plantio das mudas, com espaçamento de $3 \mathrm{~m} \times 2 \mathrm{~m}$, em covas previamente abertas de $30 \mathrm{~cm}$ x $30 \mathrm{~cm}$ x $30 \mathrm{~cm}$, que receberam 50 g de NPK 10:10:10 em cada cova. No momento do plantio, abriu-se o espaço exato para a muda com um tubete vazio acoplado em um cabo de ferramenta, a fim de não afundá-la além do colo. Após o plantio, efetuou-se irrigação localizada, duas vezes na semana, durante 60 dias. As formigascortadeiras foram controladas aplicando-se formicida granulado em seus caminhos e olhos dos formigueiros. Foi plantada uma fileira de bordadura com mudas de ipê-branco.

R. Árvore, Viçosa-MG, v.34, n.2, p.241-249, 2010
Realizaram-se cinco (05) avaliações morfológicas após o plantio das mudas em campo. Foram medidos a altura da parte aérea (a distância entre o colo e a inserção do último par de folhas no ápice das plantas), o diâmetro do coleto (com paquímetro digital STARRETâ (0-150 mm)) (NOVAES et al., 2002) e contados os números de pares de folhas verdadeiras.

As médias foram analisadas estatisticamente pelo teste de Tukey a 5\% de probabilidade, pelo programa estatístico SISVAR. Quando as médias apresentaram diferenças significativas em função das doses aplicadas, foram feitas análises de regressão, bem como suas curvas. Quando houve interação dos fatores, foram feitos os desdobramentos.

\section{RESULTADOS E DISCUSSÃO}

Os resultados estão apresentados nas Tabelas 2, 3, 4 e 5, bem como nas Figuras 1 e 2.

Houve adaptação de $100 \%$ das mudas plantadas em campo, e o preparo das covas e a irrigação, nos primeiros meses após o plantio, contribuíram para esse resultado. Houve morte de $26 \%$ das mudas, após nove (09) meses do plantio, devido à altíssima ocorrência de chuvas seguidas de altas temperaturas em fevereiro de 2006.

$\mathrm{Y}=$ altura da parte aérea $(\mathrm{cm})$; e $\mathrm{x}=$ condutividade elétrica da solução de fertirrigação (dS m $\left.{ }^{-1}\right)$.

$Y=$ height of the aerial part (cm); and $x=$ electric conductivity of fertirrigation solution ( $\left.d S \mathrm{~m}^{-1}\right)$.

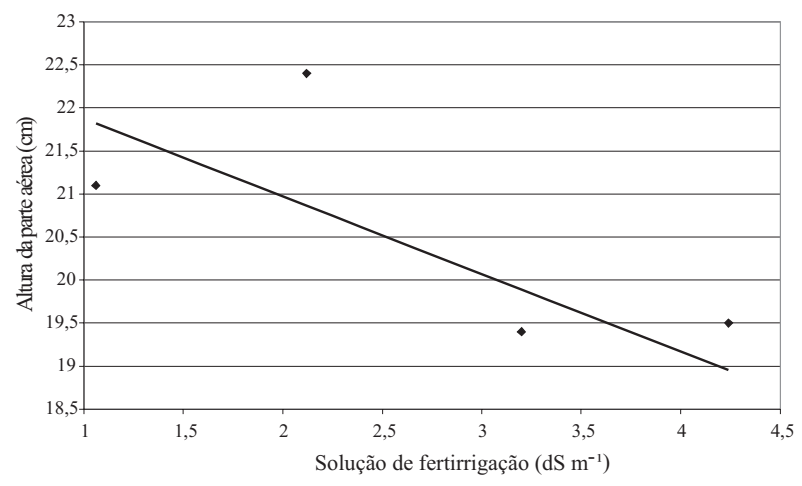

Figura 1 - Gráfico das alturas de parte aérea $(\mathrm{cm})$ de mudas de $T$ chrysotricha em função de diferentes condutividades elétricas da solução de fertirrigação usadas.

Figure 1 - Height of the aerial part (cm) of T. chrysotricha seedlings in function of different electrical conductivities of fertirrigation solution used. 
Tabela 2 - Médias obtidas nas análises de variância da altura da parte aérea (cm) de mudas de ipê-amarelo (T. chrysotricha) produzidas em diferentes substratos e diferentes soluções de fertirrigação e plantadas em campo, no Município de Taubaté, SP.

Table 2 - Means obtained in variance analyses for height of aerial part (cm) of T. chrysotricha seedlings produced in differents substrates and with differents fertirrigations solutions, and, planted in field in Taubaté, SP.

\begin{tabular}{|c|c|c|c|}
\hline \multirow[t]{2}{*}{ Fatores de variação } & 27 & 167 & 365 \\
\hline & \multicolumn{3}{|c|}{ Dias após o plantio em campo } \\
\hline Substrato (S) & $119,58^{* *}$ & $201,96^{\mathrm{NS}}$ & $3.567,38^{* *}$ \\
\hline Condutividade elétrica (CE) & $37,59^{* *}$ & $133,07^{\mathrm{NS}}$ & $517,03^{\mathrm{NS}}$ \\
\hline$S \times C E$ & $14,85^{* *}$ & $238,61^{\mathrm{NS}}$ & $1.416,68^{\mathrm{NS}}$ \\
\hline Blocos & $6,69^{\mathrm{NS}}$ & $213,30^{\mathrm{NS}}$ & $1.685,95^{\mathrm{NS}}$ \\
\hline Resíduo & 5,23 & 155,63 & 812,72 \\
\hline CV \% & 11,15 & 28,02 & 16,35 \\
\hline Média geral & 20,51 & 44,53 & 174,38 \\
\hline \multicolumn{4}{|l|}{ SUBSTRATOS - fibra de coco } \\
\hline $100 \%$ fibrosa & $17,2 \quad \mathrm{c}$ & 43,4 & $170,2 \mathrm{ab}$ \\
\hline $60 \%$ fibrosa $+40 \%$ granulada & 20,3 b & 43,9 & $167,6 \mathrm{ab}$ \\
\hline $40 \%$ fibrosa $+60 \%$ granulada & $20,8 \quad b$ & 41,3 & $161,5 \mathrm{~b}$ \\
\hline $100 \%$ granulada & 23,8 a & 49,6 & 195,8 a \\
\hline \multicolumn{4}{|l|}{ CE - SOLUÇÕES } \\
\hline $1,06 \mathrm{dS} \mathrm{m}^{-1}$ & 21,1 & 40,8 & 177,0 \\
\hline $2,12 \mathrm{dS} \mathrm{m}^{-1}$ & 22,4 & 47,6 & 166,3 \\
\hline $3,20 \mathrm{dS} \mathrm{m}^{-1}$ & 19,4 & 44,1 & 179,5 \\
\hline $4,24 \mathrm{dS} \mathrm{m}^{-1}$ & 19,5 & 45,7 & 175,6 \\
\hline \multicolumn{4}{|l|}{ REGRESSÃO } \\
\hline Equação & $Y=22,77-0,90 x$ & & \\
\hline $\mathrm{R}^{2}$ & $0,58 * *$ & & \\
\hline
\end{tabular}

$\mathrm{Y}=$ altura da parte aérea $(\mathrm{cm}) ; \mathrm{x}=$ condutividade elétrica da solução de fertirrigação $\left(\mathrm{dS} \mathrm{m}^{-1}\right) . Y=$ height of aerial part $(\mathrm{cm})$; and $\mathrm{x}=$ electric conductivity of fertirrigation solution $\left(\mathrm{dS} \mathrm{m}^{-1}\right)$.

NS - não significativo $(\mathrm{P}>0,05)$; * significativo $(\mathrm{P}<0,05)$; ** significativo $(\mathrm{P}<0,01) .{ }^{\text {NS }}$ - not significant $(\mathrm{P}>0.05)$; * significant $(P<0.05)$; and $* *$ significant $(P<0.01)$.

Médias seguidas de mesma letra na coluna não diferem entre si, pelo teste de Tukey $(\mathrm{P}>0,05)$. Means followed by the same letter in the column isn't different between them by the Tukey test $(P>0.05)$.

Houve perda de dados de duas datas de avaliação para as alturas de parte aérea, aos 54 e 102 dias após o plantio.

A última avaliação (365 dias após o plantio) contou com número menor de plantas, devido à morte das plantas aos nove (09) meses após o plantio. Como as mortes foram localizadas em manchas, houve maior perda de plantas produzidas em $60 \%$ fibrosa $+40 \%$ granulada e $40 \%$ fibrosa+ $60 \%$ granulada, porém foi possível avaliar os resultados estatisticamente com parcelas perdidas.

De forma geral, os tratamentos testados na fase de produção de mudas influenciaram no crescimento das plantas em campo (Tabela 2), principalmente em relação aos substratos testados, onde as plantas produzidas em fibra de coco $100 \%$ granulada apresentaram maiores alturas até um ano após o plantio, embora não tenha apresentado diferença significativa aos 167 dias após o plantio. Provavelmente, esses resultados são reflexos da formação do sistema radicular na fase de muda, indicando a importância da condução da formação das mudas desde a semeadura.

Verifica-se ainda, na Tabela 2, que as mudas conduzidas em diferentes soluções de fertirrigação permitiram que as plantas em campo mantivessem maiores alturas nas menores condutividades elétricas das soluções de fertirrigação (Figura 1), apenas nos primeiros meses após o plantio, igualando as alturas a partir dos 167 dias após o plantio.

Houve interação entre os fatores estudados aos 27 dias após o plantio, e os desdobramentos (Tabela 3) apontaram que as médias foram

R. Árvore, Viçosa-MG, v.34, n.2, p.241-249, 2010 
Tabela 3 - Níveis de significância e médias de altura de parte aérea $(\mathrm{cm})$ de mudas de ipê-amarelo (T. chrysotricha) plantadas em campo, no Município de Taubaté, SP, produzidas em diferentes substratos em cada solução de fertirrigação (coluna) e produzidas em cada substrato em função das soluções de fertirrigação (linha), obtidos nos desdobramentos da interação dos fatores.

Table 3 - Levels of significant and means for height of aerial part (cm) of T. chrysotricha aerial part seedlings, planted in field in Taubaté-SP, produced in different substrates in each fertirrigation solutions (column), and produced in each substrate in function of the fertirrigation solutions (line) obtained in the factors interaction.

\begin{tabular}{|c|c|c|c|c|c|c|c|}
\hline \multirow{2}{*}{$\begin{aligned} \text { FATORES } \\
\text { CE-Soluções }\end{aligned}$} & \multicolumn{7}{|c|}{27 dias após o plantio } \\
\hline & & $1,06 \mathrm{dS} \mathrm{m}^{-1}$ & $2,12 \mathrm{dS} \mathrm{m}^{-1}$ & $3,20 \mathrm{dS} \mathrm{m}^{-1}$ & $4,24 \mathrm{dS} \mathrm{m}^{-1}$ & & \\
\hline \multirow[t]{2}{*}{ 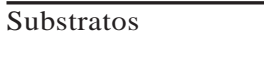 } & & ** & ** & NS & ** & Equação & $\mathrm{R}^{2}$ \\
\hline & & \multicolumn{4}{|c|}{$\mathrm{cm}-$} & & \\
\hline $100 \%$ fibrosa & * & $14,5 \quad \mathrm{c}$ & 19,9 b & 18,8 & $15,6 \quad \mathrm{c}$ & $\mathrm{Y}=6,93+9,42 \mathrm{x}-1,55 \mathrm{x}^{2}$ & $0,99 * *$ \\
\hline $\begin{array}{l}60 \% \text { fibrosa }+40 \% \\
\text { granulada }\end{array}$ & NS & 21,9 b & $22,1 \mathrm{ab}$ & 18,8 & 18,8 bc & - & \\
\hline $\begin{array}{l}40 \% \text { fibrosa }+60 \% \\
\text { granulada }\end{array}$ & Ns & $21,7 \quad b$ & $21,7 \mathrm{ab}$ & 19,8 & $19,9 \mathrm{ab}$ & - & \\
\hline $100 \%$ granulada & ** & 26,6 а & 25,8 а & 20,1 & 23,5 a & Polinomiais testad & NS \\
\hline
\end{tabular}

$\mathrm{Y}=$ altura da parte aérea $(\mathrm{cm}) ; \mathrm{x}=$ condutividade elétrica da solução de fertirrigação $\left(\mathrm{dS} \mathrm{m}^{-1}\right) . Y=$ height of the aerial part $(\mathrm{cm})$; and $\mathrm{x}=$ electric conductivity of fertirrigation solution $\left(\mathrm{dS} \mathrm{m}^{-1}\right)$.

NS - não significativo $(\mathrm{P}>0,05)$; * significativo $(\mathrm{P}<0,05)$; ** significativo $(\mathrm{P}<0,01) .{ }^{\text {NS }}$ - not significant $(\mathrm{P}>0.05)$; * significant $(P<0.05)$; and $* *$ significant $(P<0.01)$.

Médias seguidas de mesma letra na coluna não diferem entre si, pelo teste de Tukey $(\mathrm{P}>0,05)$. Means followed the same letter in the column isn't different between them by the Tukey test $(P>0.05)$.

$\mathrm{Y}=$ altura da parte aérea $(\mathrm{cm})$; e $\mathrm{x}=$ condutividade elétrica da solução de fertirrigação ( $\left.\mathrm{dS} \mathrm{m}^{-1}\right)$.

$\mathrm{Y}=$ height of the aerial part $(\mathrm{cm})$; and $\mathrm{x}=$ electric conductivity of fertirrigation solution ( $\mathrm{dS} \mathrm{m}^{-1}$ ).

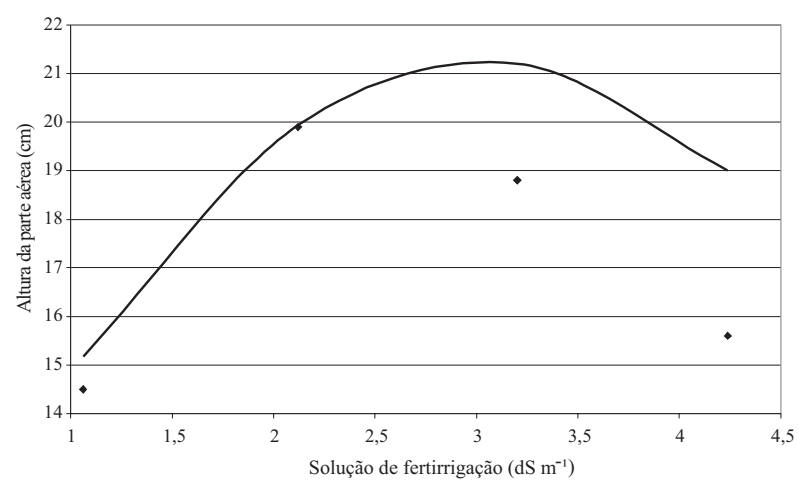

Figura 2-Gráfico das alturas de parte aérea $(\mathrm{cm})$ de mudas de T chrysotricha produzidas em fibra de coco $100 \%$ fibrosa, em função de diferentes condutividades elétricas da solução de fertirrigação usadas, quando plantadas em campo.

Figure 2 - Height of the aerial part (cm) of T. chrysotricha seedlings produced in coconut fiber $100 \%$ fibrous, in function of different electrical conductivities of fertirrigation solution used, when planted in a field. estatisticamente diferentes entre os substratos de produção para mudas produzidas em cada solução de fertirrigação, exceto em solução de condutividade elétrica de 3,2 dS m¹. Mudas conduzidas em fibra de coco $100 \%$ granulada alcançaram maiores alturas nessa época de avaliação, reflexo do encontrado anteriormente em viveiro.

Plantas formadas em fibra de coco $100 \%$ fibrosa alcançaram maiores médias quando produzidas em solução de 2,12 dS m¹ (Figura 2). As médias das alturas de mudas formadas em substrato $100 \%$ fibrosa ajustaram-se em curva de regressão quadrática em função das condutividades elétricas das soluções de fertirrigação. As mudas produzidas em 100\% granulada não ajustaram suas médias nas regressões polinomiais testadas, porém apresentaram tendência de maiores médias nas mudas conduzidas em menores condutividade elétrica das soluções de fertirrigação.

Os diâmetros de coleto estão apresentados na Tabela 4. De forma geral, os diâmetros aumentaram bem lentamente após o plantio, e até os 102 dias após o plantio houve acréscimos mínimos de apenas 0,06 cm em média. 
Tabela 4-Médias obtidas nas análises de variância do diâmetro do coleto (cm) de mudas de ipê-amarelo (T. chrysotricha) produzidas em diferentes substratos e diferentes soluções de fertirrigação e plantadas em campo, no Município de Taubaté, SP.

Table 4 - Means obtained in variance analyses for stem diameter $(\mathrm{cm})$ of $\mathbf{T}$. chrysotricha seedlings produced in differents substrates and with differents fertirrigations solutions, and planted in field in Taubaté, SP.

\begin{tabular}{|c|c|c|c|c|c|}
\hline \multirow[t]{2}{*}{ Fatores de variação } & 27 & 54 & 102 & 167 & 365 \\
\hline & \multicolumn{5}{|c|}{ Dias após o plantio em campo } \\
\hline$\overline{\text { Substrato (S) }}$ & $0,02^{* *}$ & $0,02^{* *}$ & $0,030^{* *}$ & $0,03^{\mathrm{NS}}$ & $0,23^{\mathrm{NS}}$ \\
\hline Condutividade elétrica (CE) & $0,003^{* *}$ & $0,001^{* *}$ & $0,005^{* *}$ & $0,04^{* *}$ & $0,01^{* *}$ \\
\hline $\mathrm{S} \times \mathrm{CE}$ & $0,001^{\mathrm{NS}}$ & $0,001^{\mathrm{NS}}$ & $0,001^{\mathrm{NS}}$ & $0,03^{\mathrm{NS}}$ & $0,25^{\mathrm{NS}}$ \\
\hline Blocos & $0,0015^{\mathrm{NS}}$ & $0,001^{\mathrm{NS}}$ & $0,002^{\mathrm{NS}}$ & $0,02^{\mathrm{NS}}$ & $0,14^{\mathrm{NS}}$ \\
\hline Resíduo & 0,001 & 0,001 & 0,002 & 0,02 & 0,15 \\
\hline $\mathrm{CV} \%$ & 10,03 & 8,70 & 10,61 & 18,70 & 15,52 \\
\hline Média geral & 0,36 & 0,37 & 0,42 & 0,75 & 2,46 \\
\hline \multicolumn{6}{|l|}{ SUBSTRATOS - fibra de coco } \\
\hline $100 \%$ fibrosa & $0,31 \quad c$ & 0,33 & $0,38 \quad b$ & 0,74 & 2,33 \\
\hline $60 \%$ fibrosa $+40 \%$ granulada & $0,35 \mathrm{~b}$ & $0,36 \mathrm{~b}$ & $0,41 \quad b$ & 0,74 & 2,52 \\
\hline $40 \%$ fibrosa $+60 \%$ granulada & $0,37 \mathrm{ab}$ & $0,38 \mathrm{~b}$ & $0,42 \quad b$ & 0,70 & 2,41 \\
\hline $100 \%$ granulada & 0,39 a & 0,41 a & 0,48 a & 0,80 & 2,61 \\
\hline \multicolumn{6}{|l|}{ CE - SOLUÇÕES } \\
\hline $1,06 \mathrm{dS} \mathrm{m}^{-1}$ & 0,34 & 0,36 & 0,41 & 0,68 & 2,43 \\
\hline $2,12 \mathrm{dS} \mathrm{m}^{-1}$ & 0,36 & 0,37 & 0,43 & 0,77 & 2,48 \\
\hline $3,20 \mathrm{dS} \mathrm{m}^{-1}$ & 0,35 & 0,37 & 0,41 & 0,73 & 2,49 \\
\hline $4,24 \mathrm{dS} \mathrm{m}^{-1}$ & 0,38 & 0,38 & 0,45 & 0,79 & 2,44 \\
\hline
\end{tabular}

Ns - não significativo $(\mathrm{P}>0,05)$; * significativo $(\mathrm{P}<0,05)$; ** significativo $(\mathrm{P}<0,01) .{ }^{\text {NS }}-$ not significant $(P>0.05)$; * significant $(P<0.05)$; ** significant $(P<0.01)$.

Médias seguidas de mesma letra na coluna não diferem entre si, pelo teste de Tukey $(\mathrm{P}>0,05)$. Means followed by the same letter in the column aren't different between them by the Tukey test $(P>0.05)$.

As médias de diâmetros de coleto (Tabela 4) diferiram entre si em função dos substratos testados aos 27, 54 e 102 dias após o plantio, quando, maiores valores foram encontrados nas plantas conduzidas em fibra de coco 100\% granulada na fase de muda, semelhante ao encontrado nas avaliações de viveiro. Nas demais épocas de avaliação, embora não tenham apresentado diferenças estatisticamente diferentes, maiores médias foram encontradas nas plantas produzidas em $100 \%$ granulada, inclusive após um ano do plantio. Esses dados se tornam importantíssimos, pois os resultados encontrados em viveiro persistiram após um ano da data do plantio em campo.

Os diâmetros de coleto de plantas de ipê-amarelo, produzidas na fase de muda em soluções de adubação com diferentes condutividades elétricas, mostraram-se muito próximos após o plantio em campo.

O número de pares de folhas foi menor no início das avaliações de campo (Tabela 5) em relação ao final das avaliações de viveiro, segundo Sarzi et al. (2008) possivelmente por queda de folhas. O acréscimo desse número, assim como o diâmetro, foi lento, dobrando apenas aos 167 dias após o plantio. Houve aumento bem significativo no segundo semestre após o plantio, coincidindo com maiores temperaturas no verão.

O número de pares de folhas de ipê-amarelo plantado em campo no Município de Taubaté (Tabela 5) apresentou diferença significativa em função dos substratos de produção aos 167 dias após o plantio, quando as plantas produzidas em fibra de coco $100 \%$ granulada apresentaram mais folhas, não diferindo estatisticamente dos substratos $60 \%$ fibrosa $+40 \%$ granulada e $40 \%$ fibrosa $+60 \%$ granulada.

Esses resultados indicam que as mudas conduzidas em fibra de coco $100 \%$ granulada foram mais vigorosas em relação às demais, pois, embora não tenha havido diferenças estatisticamente diferentes, em todas as épocas amostradas após o plantio, essas plantas se encontraram mais altas (Tabela 2), com maiores diâmetro de coleto (Tabela 4) e maiores números de folhas (Tabela 5).

R. Árvore, Viçosa-MG, v.34, n.2, p.241-249, 2010 
Tabela 5 - Médias obtidas nas análises de variância do número de pares de folhas de mudas de ipê-amarelo (T. chrysotricha) produzidas em diferentes substratos e diferentes soluções de fertirrigação e plantadas em campo, no Município de Taubaté, SP.

Table 5 - Means obtained in variance analyses for leaves pairs number of T. chrysotricha seedlings produced in differents substrates and with differents fertirrigations solutions, and planted in field in Taubaté, SP.

\begin{tabular}{|c|c|c|c|c|c|}
\hline Fatores de variação & 27 & 54 & 102 & 167 & 365 \\
\hline & \multicolumn{5}{|c|}{ Dias após o plantio em campo } \\
\hline Substrato (S) & $7,01^{\mathrm{NS}}$ & $3,94^{\mathrm{NS}}$ & $17,66^{\mathrm{NS}}$ & $39,96^{* *}$ & $1.556,78^{\mathrm{NS}}$ \\
\hline Condutividade elétrica (CE) & $4,91^{\mathrm{NS}}$ & $2,57^{\mathrm{NS}}$ & $4,61^{\mathrm{NS}}$ & $1,89^{\mathrm{NS}}$ & $1.181,37^{\mathrm{NS}}$ \\
\hline $\mathrm{S} \times \mathrm{CE}$ & $5,33^{\mathrm{NS}}$ & $1,93^{\mathrm{NS}}$ & $5,15^{\mathrm{NS}}$ & $19,20^{\mathrm{NS}}$ & $745,18^{\mathrm{NS}}$ \\
\hline Blocos & $6,81^{\mathrm{NS}}$ & $1,03^{\mathrm{NS}}$ & $10,53^{\mathrm{NS}}$ & $20,43^{\mathrm{NS}}$ & $1.106,70^{\mathrm{NS}}$ \\
\hline Resíduo & 3,45 & 2,18 & 5,75 & 10,16 & 551,12 \\
\hline CV \% & 30,30 & 25,20 & 30,27 & 19,24 & 27,09 \\
\hline Média geral & 6 & 6 & 8 & 17 & 87 \\
\hline \multicolumn{6}{|l|}{ SUBSTRATOS - fibra de сосо } \\
\hline $100 \%$ fibrosa & 5 & 5 & 7 & $15 \mathrm{~b}$ & 81 \\
\hline $60 \%$ fibrosa $+40 \%$ granulada & 6 & 6 & 8 & $16 \mathrm{ab}$ & 78 \\
\hline $40 \%$ fibrosa $+60 \%$ granulada & 7 & 6 & 7 & $16 a b$ & 85 \\
\hline $100 \%$ granulada & 7 & 7 & 9 & 19 a & 101 \\
\hline \multicolumn{6}{|l|}{ CE - SOLUÇÕES } \\
\hline $1,06 \mathrm{dS} \mathrm{m}^{-1}$ & 7 & 6 & 7 & 16 & 79 \\
\hline $2,12 \mathrm{dS} \mathrm{m}^{-1}$ & 6 & 6 & 9 & 17 & 78 \\
\hline $3,20 \mathrm{dS} \mathrm{m}^{-1}$ & 5 & 6 & 8 & 16 & 94 \\
\hline $4,24 \mathrm{dS}^{-1}$ & 6 & 6 & 8 & 17 & 94 \\
\hline
\end{tabular}

NS - não significativo $(\mathrm{P}>0,05)$; * significativo $(\mathrm{P}<0,05)$; $\mathrm{e}^{* *}$ significativo $(\mathrm{P}<0,01) .{ }^{\text {NS }}$ - not significant $(P>0.05)$; * significant $(P<0.05)$; and $* *$ significant $(P<0.01)$.

Médias seguidas de mesma letra na coluna não diferem entre si, pelo teste de Tukey $(\mathrm{P}>0,05)$. Means followed by the same letter in the column aren't different between them by Tukey test $(P>0.05)$.

Em relação às condutividades elétricas das soluções de adubação utilizadas na produção das mudas de ipêamarelo, nota-se, pela Tabela 5, que não influenciaram estatisticamente no número de pares de folhas nas épocas amostradas após o plantio em campo. Houve tendência de apresentarem mais folhas as mudas produzidas nas maiores condutividades elétricas aos 365 dias após o plantio.

\section{CONCLUSÃO}

Considerando as condições deste experimento e os tratamentos testados, conclui-se que:

- Aadaptação em campo de mudas de T. chrysotricha independe de substratos e soluções de fertirrigação.

- Mudas de T. chrysotricha produzidas em fibra de coco $100 \%$ granulada alcançam maiores alturas, diâmetros de coleto e números de pares de folhas quando plantadas em campo.

- As condutividades elétricas das soluções de fertirrigação testadas não influenciam no desenvolvimento de mudas de $T$. chrysotricha quando plantadas em campo.

\section{AGRADECIMENTOS}

À Amafibra do grupo Sococo - fibras e substratos agrícolas da Amazônia.

\section{Ao Viveiro Florestal Câmara.}

Ao Curso de Pós-Graduação em Agronomia, área de concentração em Horticultura, da Faculdade de Ciências Agronômicas da UNESP de Botucatu, SP.

Aos Diretores do Instituto Florestal da Secretaria de Estado do Meio Ambiente.

Aos estagiários da Seção de Silvicultura, em especial Luanda Janucci Marino.

Aos funcionários do Viveiro Florestal de Taubaté.

\section{REFERÊNCIAS}

CARVALHO FILHO, J. L. S. et al. Produção de mudas de jatobá (Hymenaea courbaril L.) em diferentes ambientes, recipientes e composições de substratos. Cerne, v.9, n.1, p.109-118, 2003. 
CRUZ, C. A. F. e et al. Efeito de diferentes níveis de saturação por bases no desenvolvimento e qualidade de ipê-roxo (Tabebuia impetiginosa (Mart.) Standley). Scientia Forestalis, n.66, p.100-107, 2004.

GONÇALVES, J. L. M. et al. Produção de mudas de espécies nativas: substrato, nutrição, sombreamento e fertilização. In: GOLÇALVES, J. L. M.; BENEDETTI, V. (Orgs.). Nutrição e fertilização florestal. Piracicaba: 2005. p.309-350.

LEITE, H. G. et al. Determinação dos custos da qualidade em produção de mudas de eucalipto. Revista Árvore, v.29, n.6, p.955-964, 2005.

\section{LOPES, J. L. W. Efeitos de diferentes} substratos na qualidade e no ciclo de produção de mudas de Pinus caribaea Morelet var. hondurensis: 2002. 76f.

Especialização (Engenharia de Produção) Universidade Estadual Paulista, Bauru, 2002.

LORENZI, H. Árvores brasileiras: manual de identificação e cultivo de plantas arbóreas nativas do Brasil. Nova Odessa: Plantarum, 1992. 352p.

MAIA, C. M. B. F. Uso de casca de Pinus e lodo biológico com substrato para produção de mudas de Pinus taeda. Boletim de Pesquisa Florestal, n.39, p.81-92, 1999.
MORAES NETO, S. P. et al. Fertilização de mudas de espécies arbóreas nativas e exóticas. Revista Árvore, v.27, n.2, p.129-137, 2003.

NOVAES, A. B. et al. Avaliação do potencial de regeneração de raízes de mudas de Pinus taeda L., produzidas em diferentes tipos de recipientes, e o seu desempenho no campo. Revista Árvore, v.26, n. 6, p.675-681, 2002.

PAULINO, A. F. et al. Distribuição do sistema radicular de árvores de acácia-negra oriundas de mudas produzidas em diferentes recipientes. Revista Árvore, v.27, n.5, p.605-610, 2003.

RESENDE, A. V. et al. Acúmulo e eficiência nutricional de macronutrientes por espécies florestais de diferentes grupos sucessionais em resposta à fertilização fosfatada. Ciência Agrotécnica, v.24, n.1, p.160-173, 2000.

SARZI, I.; VILLAS BÔAS, R. L.; SILVA, M. R. Desenvolvimento de mudas de Tabebuia chrysotricha em função de substratos e de soluções de fertirrigação. Revista Cerne, v.14, n.2, p.153-162, 2008. 